\title{
Change in Clinicopathological Characteristics of Patients With Colorectal Cancer During the COVID- 19 Epidemic in China: a Single-institution Retrospective Analysis Based on Real-world Data
}

Yu Yang

Chinese PLA General Hospital

Yuxuan Li

Chinese PLA General Hospital

Songyan Li

Chinese PLA General Hospital

Xiaohui Du ( $\sim$ duxiaohui301@sina.com )

Chinese PLA General Hospital https://orcid.org/0000-0002-8713-1358

Research article

Keywords: Colorectal cancer surgery, Coronavirus disease 2019, Clinical characters

Posted Date: January 15th, 2021

DOI: https://doi.org/10.21203/rs.3.rs-145239/v1

License: (c) (1) This work is licensed under a Creative Commons Attribution 4.0 International License.

Read Full License 


\section{Abstract}

Background: The coronavirus disease 2019 is in the global epidemic, which seriously affects the normal medical order. How to effectively manage colorectal cancer under the background of regular epidemic prevention and control need to gather the experience and wisdom of countries all over the world to study together.

Methods: A retrospective observational study was performed to compare colorectal cancer patients' clinical characters between the severe epidemic group (SEG,January 20, 2020 to March 20, 2020) versus the epidemic remission group (ERG, April 20, 2019 to June 20, 2020). The demographic characteristics, surgical data, postoperative pathological features and postoperative clinical characteristics of the two groups were compared.

Results: 112 patients were enrolled in the study, including 47 patients in SEG and 65 patients in ERG. There was no significant difference in age, sex, height, weight and body mass index between two groups. The proportion of local patients in SEG was significantly higher than that in ERG $(P=0.001)$. More patients in ERG received preoperative neoadjuvant chemoradiotherapy (Neo-CRT) $(P=0.025)$. There was no significant difference in $\mathrm{pT}$ staging, $\mathrm{pN}$ staging and $\mathrm{pM}$ staging between the two groups $(P>0.05)$, but the overall pTNM staging was earlier in the SEG $(P<0.001)$. At the same time, the proportion of vascular invasion $(P=0.004)$ and perineural invasion $(P<0.001)$ in SEG was higher than that in ERG. There were no postoperative complications in STG group, but 6 patients in ECG group had postoperative complications, including 4 cases of incision infection and 2 cases of anastomotic leakage. It should be noted that, 5 of these 6 patients received Neo-CRT before operation.

Conclusion: our study shows that due to the interference of serious epidemic situation to medical activities and more patients choosing preoperative neoadjuvant chemoradiotherapy, some patients delayed operation to the period of the epidemic remission, which may lead to the progress of tumor staging and increase the risk of postoperative complications. In addition, we hope that our strategy for colorectal cancer diagnosis and treatment during the epidemic will help other surgeons.

\section{Background}

The first case of Coronavirus disease 2019 (COVID-19) was diagnosed in Wuhan, China in December 2019, and then COVID-19 broke out across the country and gradually spread to countries around the world[1]. On February 12, 2020, the International Committee on Taxonomy of Viruses (ICTV) officially named COVID-19 pathogen 2019-nCoV. So far, the host and intermediate host of 2019-nCoV are not clear, and its infectivity is very strong, through the epidemiological study, the Basic reproduction number (R0) of 2019-nCoV is 2.2, which is generally susceptible to the population[2]. On January 20, 2020, the National Health Commission of China included COVID-19 into Class B infectious diseases and carried out prevention and treatment according to Class $\mathrm{A}$ infectious diseases. A highly contagious and widespread epidemic like COVID-19 is rare, most clinicians lack experience in how to carry out clinical work normally 
under such circumstances. At present, we are still unable to make an accurate judgment on when the epidemic will end. Therefore, it is an arduous task to provide the best medical services for patients on the premise of protecting the safety of patients, their families and medical workers.

Cancer treatment is a long process, and tumor patients are at high risk of being infected with COVID-19. According to the study of He et al, the proportion of tumor patients in COVID-19 is higher than that in the general population. Secondly, compared with non-tumor patients, COVID-19 infected tumor patients have older age, more obvious respiratory symptoms, higher baseline pulmonary CT severity and higher severe risk. Tumor history is the highest risk factor for serious events[3]. What we need to be more vigilant about is that patients who have received chemotherapy or surgery in the near future have a higher risk of severe illness than those who have not started antineoplastic therapy. In addition, the hospital as a high-risk place for infection, tumor patients who come for treatment also have to face the risk of cross-infection[4]. Nevertheless, radical surgery is indispensable for prolonging the survival of most patients with colorectal cancer (CRC). Therefore, this requires us to make a reasonable clinical decision on the risk of infection faced by patients undergoing hospitalization surgery and the disease progression caused by delayed treatment.

With the relief of the COVID-19 epidemic in China, the daily number of new cases in most parts of China is single digit or zero, various industries are gradually returning to work, and the government has proposed to effectively protect the medical needs of non-COVID-19 patients. Our hospital has resumed its daily work since March 2020, and the volume of diagnosis and treatment has been adjusted day by day according to the changes in epidemic prevention and control requirements. In this paper, the clinical data of CRC patients in the two periods of severe epidemic situation and mild epidemic situation were reviewed and analyzed, and the differences of clinicopathological characteristics between the two groups were compared. At the same time, the surgical diagnosis and treatment strategies for CRC patients during the epidemic situation in our center are introduced,hoping to benefit other surgeons.

\section{Methods}

\section{Study Design and Patients}

The Beijing municipal people's government officially announced the first confirmed case of COVID-19 on January 20, 2020, and with zero new cases on March 20, 2020 for the first time. The General Hospital of the Chinese people's Liberation Army (Beijing) has strengthened the management of COVID-19 infection cases since January 20,2020, including outpatient, hospitalization and discharge management. After March 20, 2020, the epidemic situation of COVID-19 began to stabilize and his hospitalization gradually recovered. Therefore, we believe that the severe period of the epidemic is from January 20, 2020 to March 20, 2020, and after March 20, 2020 is the period of remission of the epidemic. According to hospitalization date, the patients were divided into severe epidemic group (SEG, January 20, 2020 to March 20, 2020) and epidemic remission group (ERG, April 20, 2020 to June 20, 2020). 
The clinical data of CRC patients in general surgery of our hospital were collected, and the inclusion criteria were as follows: (1) all patients received surgical treatment, including radical surgery and other non-radical operations for CRC; (2) the operations were performed by the same team of experienced surgeons; (3) all patients with CRC were diagnosed by pathological examination and all clinical data were integral and available. Exclusion criteria: patients who underwent emergency surgery were excluded. All patients signed the informed consent form before operation, and this study was approved by the Ethics Committee of Chinese PLA General Hospital.

\section{Data collection}

This study is a retrospective study, and the observation indicators include demographic information (sex, age, source, etc.), surgical information (tumor location, operation time, intraoperative bleeding, etc.), pathological information (tumor stage, immunohistochemical results, etc.). postoperative clinical information (days of hospitalization, etc.).

\section{Statistical analysis}

Statistical analysis was performed using SPSS 26.0 (IBM, Armonk, New York, United States). The measurement data were presented with mean \pm standard deviation $(x \pm s)$, t-test was used for inter-group comparison; the enumeration data were presented with $\mathrm{n}(\%)$, and Chi-square test or Fisher's exact test was used for inter-group comparison. $P<0.05$ indicates that the difference is statistically significant.

\section{Results}

\section{Baseline demographical data}

112 patients were enrolled in the study, including 47 patients in SEG and 65 patients in ERG. The demographic characteristics of the two groups are shown in Table 1. There was no significant difference in age, sex, height, weight and body mass index (BMI) between SEG and ERG. There was a significant difference in origin of patients between the two groups, and the proportion of local patients in SEG was significantly higher than that in ERG $(P=0.001)$. More patients in ERG received neoadjuvant chemoradiotherapy (Neo-CRT) before operation $(P=0.025)$ (Table 1$)$. 
Table 1

Baseline demographical data of all patients enrolled

\begin{tabular}{|c|c|c|c|}
\hline \multirow[t]{2}{*}{ Demographics } & SEG $(n=47)$ & ECG $(n=65)$ & \multirow[t]{2}{*}{$P$} \\
\hline & $\mathrm{M} \pm \mathrm{SD}$ or $\mathrm{N}(\%)$ & $\mathrm{M} \pm \mathrm{SD}$ or $\mathrm{N}(\%)$ & \\
\hline Age(years) & 61.6(10.9) & $59.1(11.7)$ & 0.730 \\
\hline Sex & & & 0.186 \\
\hline Male & $30(63.8)$ & 49(75.4) & \\
\hline Female & 17(36.2) & $16(24.6)$ & \\
\hline Height(cm) & 167.2(8.3) & 168.3(7.2) & 0.100 \\
\hline Weight(kg) & $68.8(12.5)$ & $70.4(12.8)$ & 0.252 \\
\hline $\mathrm{BMI}\left(\mathrm{kg} / \mathrm{m}^{2}\right)$ & & & 0.897 \\
\hline$<24$ & 23(48.9) & $31(47.7)$ & \\
\hline$\geq 24$ & $24(51.1)$ & $34(52.3)$ & \\
\hline Neo-CRT & & & 0.025 \\
\hline Yes & $2(4.3)$ & 12(18.5) & \\
\hline No & $45(95.7)$ & $53(81.5)$ & \\
\hline Origin of patients & & & 0.001 \\
\hline Local & $26(55.3)$ & $16(24.6)$ & \\
\hline Nonlocal & $21(44.7)$ & 49(75.4) & \\
\hline
\end{tabular}

\section{Clinicopathological data}

The proportion of rectal cancer and ascending colon cancer was higher in the SEG group $(P=0.017)$, and there were no statistically significant differences in terms of surgery time, operative method and transfusion of blood $(P>0.05)$ (Table 2). 
Table 2

Intraoperative clinicopathological data of all patients enrolled

\begin{tabular}{|c|c|c|c|}
\hline \multirow[t]{2}{*}{ Clinicopathological data } & $\operatorname{SEG}(n=47)$ & $\operatorname{ECG}(n=65)$ & \multirow[t]{2}{*}{$P$} \\
\hline & $\mathrm{M} \pm \mathrm{SD}$ or $\mathrm{N}(\%)$ & $\mathrm{M} \pm \mathrm{SD}$ or $\mathrm{N}(\%)$ & \\
\hline Cancer localization & & & 0.017 \\
\hline Cecum & $0(0)$ & $3(4.6)$ & \\
\hline Ascending colon & $11(23.4)$ & $8(12.3)$ & \\
\hline Transverse colon & $0(0)$ & $0(0)$ & \\
\hline Descending colon & $2(4.3)$ & $1(1.5)$ & \\
\hline Sigmoid colon & $4(8.5)$ & $20(30.8)$ & \\
\hline Rectum & $30(63.8)$ & $33(50.8)$ & \\
\hline Operative method & & & 0.953 \\
\hline Open & $9(19.1)$ & 11(16.9) & \\
\hline Laparoscopic & $36(76.6)$ & $51(78.5)$ & \\
\hline Conversion to open & $2(4.3)$ & $3(4.6)$ & \\
\hline Surgery time(min) & $188.68(68.2)$ & 159.5(58.2) & 0.252 \\
\hline Transfusion of blood & & & 0.458 \\
\hline Yes & $2(4.3)$ & $5(7.7)$ & \\
\hline No & $45(95.7)$ & $60(92.3)$ & \\
\hline pT stage & & & 0.123 \\
\hline T1 & $3(6.4)$ & $2(3.1)$ & \\
\hline T2 & $10(21.3)$ & $7(10.8)$ & \\
\hline T3 & $19(40.4)$ & $35(53.8)$ & \\
\hline $\mathrm{T} 4 \mathrm{a}$ & $14(29.8)$ & $14(21.5)$ & \\
\hline $\mathrm{T} 4 \mathrm{~b}$ & $1(2.1)$ & $7(10.8)$ & \\
\hline pN stage & & & 0.123 \\
\hline NO & $35(74.5)$ & $34(52.3)$ & \\
\hline N1a & $6(12.8)$ & $11(16.9)$ & \\
\hline
\end{tabular}

ECG, epidemic remission group; $M$, mean; N, number; SEG, severe epidemic group; SD, standard deviation. 


\begin{tabular}{|c|c|c|c|}
\hline \multirow[t]{2}{*}{ Clinicopathological data } & $\operatorname{SEG}(n=47)$ & $\operatorname{ECG}(n=65)$ & \multirow[t]{2}{*}{$P$} \\
\hline & $M \pm S D$ or $N(\%)$ & $M \pm S D$ or $N(\%)$ & \\
\hline N1b & $4(8.5)$ & $8(12.3)$ & \\
\hline N2a & $1(2.1)$ & $6(9.2)$ & \\
\hline $\mathrm{N} 2 \mathrm{~b}$ & $1(2.1)$ & $6(9.2)$ & \\
\hline pM stage & & & 0.643 \\
\hline M0 & $44(93.6)$ & 61(93.8) & \\
\hline M1a & $3(6.4)$ & $3(4.6)$ & \\
\hline M1b & $0(0)$ & $1(1.5)$ & \\
\hline pTNM stage & & & $<0.001$ \\
\hline Stage $\otimes$ & $12(25.5)$ & $2(3.1)$ & \\
\hline Stage $\mathbb{Z}$ & $20(42.6)$ & $12(18.5)$ & \\
\hline Stage $\mathbb{Z}$ & $12(25.5)$ & 47(72.3) & \\
\hline Stage $\mathbb{Z}$ & $3(6.4)$ & $4(6.2)$ & \\
\hline Differentiation & & & 0.711 \\
\hline Poor & $10(21.3)$ & 12(18.5) & \\
\hline Moderate & $37(78.7)$ & $53(81.5)$ & \\
\hline Well & $0(0)$ & $0(0)$ & \\
\hline Length of tumor(cm) & $4.5(1.9)$ & $4.8(1.9)$ & 0.702 \\
\hline Histological type & & & 0.077 \\
\hline Adenocarcinoma & $37(78.7)$ & $56(86.2)$ & \\
\hline Mucinous adenocarcinoma & 10(21.3) & $6(9.2)$ & \\
\hline Signet-ring cell carcinoma & $0(0)$ & $3(4.6)$ & \\
\hline Vascular invasion & & & 0.004 \\
\hline Yes & $12(25.5)$ & $4(6.2)$ & \\
\hline No & $35(74.5)$ & $61(93.8)$ & \\
\hline Perineural invasion & & & $<0.001$ \\
\hline
\end{tabular}

ECG, epidemic remission group; $M$, mean; N, number; SEG, severe epidemic group; SD, standard deviation. 


\begin{tabular}{|c|c|c|c|}
\hline \multirow[t]{2}{*}{ Clinicopathological data } & SEG $(n=47)$ & $\operatorname{ECG}(n=65)$ & \multirow[t]{2}{*}{$P$} \\
\hline & $\mathrm{M} \pm \mathrm{SD}$ or $\mathrm{N}(\%)$ & $\mathrm{M} \pm \mathrm{SD}$ or $\mathrm{N}(\%)$ & \\
\hline Yes & $9(19.1)$ & $0(0)$ & \\
\hline No & $38(80.9)$ & $65(100)$ & \\
\hline \multicolumn{2}{|l|}{ Tumor deposit } & & 0.942 \\
\hline Yes & $6(12.8)$ & $8(12.3)$ & \\
\hline No & 41(87.2) & $57(87.7)$ & \\
\hline $\begin{array}{l}\text { ECG, epidemic remission } \\
\text { deviation. }\end{array}$ & ; N, number; SEG & epidemic group; & idard \\
\hline
\end{tabular}

There was no significant difference in $\mathrm{pT}$ staging, $\mathrm{pN}$ staging and $\mathrm{pM}$ staging between the two groups $(P$ $>0.05)$, but the overall pTNM staging was earlier in the SEG $(P<0.001)$. The proportion of patients with

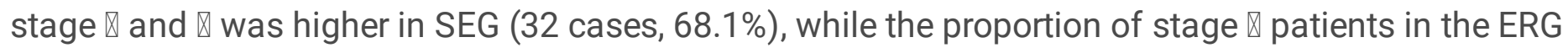
was higher (47 cases, $72.3 \%)$. At the same time, the proportion of vascular invasion $(P=0.004)$ and perineural invasion $(P<0.001)$ in SEG was higher than that in ERG. There was no significant difference in tumor histological type, differentiation, length of tumor and tumor deposit between the two groups $(P>$ 0.05) (Table 3). 
Table 3

Immunohistochemical data of all patients enrolled

\begin{tabular}{|c|c|c|c|}
\hline \multirow[t]{2}{*}{ Immunohistochemica data } & $\operatorname{SEG}(n=47)$ & $\operatorname{ECG}(n=65)$ & \multirow[t]{2}{*}{$P$} \\
\hline & $\mathrm{M} \pm \mathrm{SD}$ or $\mathrm{N}(\%)$ & $\mathrm{M} \pm \mathrm{SD}$ or $\mathrm{N}(\%)$ & \\
\hline Ki67(\%) & $76.17(13.6)$ & 76.31(14.6) & 0.975 \\
\hline HER1 & & & 0.135 \\
\hline positive & $47(100)$ & $62(95.4)$ & \\
\hline negative & $0(0)$ & $3(4.6)$ & \\
\hline HER2 & & & 0.106 \\
\hline positive & $37(78.7)$ & $42(64.6)$ & \\
\hline negative & 10(21.3) & 23(35.4) & \\
\hline KRAS & & & 0.108 \\
\hline positive & $23(48.9)$ & 22(33.8) & \\
\hline negative & $24(51.1)$ & $43(66.2)$ & \\
\hline NRAS & & & 0.135 \\
\hline positive & $0(0)$ & $3(4.6)$ & \\
\hline negative & $47(100)$ & $62(95.4)$ & \\
\hline BRAF & & & 0.237 \\
\hline positive & $1(2.1)$ & $0(0)$ & \\
\hline negative & $46(97.9)$ & $65(100)$ & \\
\hline PIK3CA & & & 0.237 \\
\hline positive & $1(2.1)$ & $0(0)$ & \\
\hline negative & $46(97.9)$ & $65(100)$ & \\
\hline MLH1 & & & 0.740 \\
\hline positive & $45(95.7)$ & 63(96.9) & \\
\hline negative & $2(4.3)$ & $2(3.1)$ & \\
\hline $\mathrm{MSH} 2$ & & & 0.759 \\
\hline positive & 46(97.9) & $63(96.9)$ & \\
\hline
\end{tabular}

dMMR, deficient mismatch repair; ECG, epidemic remission group; $M$, mean; $N$, number; pMMR, proficient mismatch repair; SEG, severe epidemic group; SD, standard deviation. 


\begin{tabular}{|c|c|c|c|}
\hline \multirow[t]{2}{*}{ Immunohistochemica data } & $\operatorname{SEG}(n=47)$ & $\operatorname{ECG}(n=65)$ & \multirow[t]{2}{*}{$P$} \\
\hline & $\mathrm{M} \pm \mathrm{SD}$ or $\mathrm{N}(\%)$ & $\mathrm{M} \pm \mathrm{SD}$ or $\mathrm{N}(\%)$ & \\
\hline negative & $1(2.1)$ & $2(3.1)$ & \\
\hline MSH6 & & & 0.379 \\
\hline positive & 45(95.7) & $64(98.5)$ & \\
\hline negative & $2(4.3)$ & $1(1.5)$ & \\
\hline PMS2 & & & 0.740 \\
\hline positive & $45(95.7)$ & 63(96.9) & \\
\hline negative & $2(4.3)$ & $2(3.1)$ & \\
\hline Mismatch repair & & & 0.682 \\
\hline pMMR & $44(93.6)$ & $62(95.4)$ & \\
\hline dMMR & $3(6.4)$ & $3(4.6)$ & \\
\hline
\end{tabular}

Immunohistochemical examination of tumor tissue showed that there was no significant difference in Ki67, HER1, HER2, KRAS, NRAS, BRAF between the two groups $(P>0.05)$. In terms of DNA mismatch repair function index reflecting tumor microsatellite stability, there was no significant difference in MLH1, MSH2, MSH6 and PMS2, while, the proportion of deficient mismatch repair (dMMR) between the two groups $(P>0.05)$ (Table 3$)$.

\section{Postoperative clinical data}

There were no postoperative complications in STG group, but 6 patients in ECG group had postoperative complications, including 4 cases of incision infection and 2 cases of anastomotic leakage. It should be noted that, 5 of these 6 patients received Neo-CRT before operation. There was no significant difference in total hospital stays between the two groups $(P>0.05)$ (Table 4$)$. 
Table 4

Postoperative clinical data of all patients enrolled

\begin{tabular}{|c|c|c|c|}
\hline \multirow[t]{2}{*}{ Postoperative clinical data } & SEG $(n=47)$ & $\operatorname{ECG}(n=65)$ & \multirow[t]{2}{*}{$P$} \\
\hline & $M \pm S D$ or $N(\%)$ & $\mathrm{M} \pm \mathrm{SD}$ or $\mathrm{N}(\%)$ & \\
\hline Complication & & & 0.032 \\
\hline Yes & $0(0)$ & $6(9.2)$ & \\
\hline No & $47(100)$ & $59(90.8)$ & \\
\hline Total hospital stays(day) & 13.6(3.4) & $11.5(3.6)$ & 0.596 \\
\hline
\end{tabular}

\section{Discussion}

Colorectal cancer (CRC) is one of the most common malignant tumors, with more than 1.8 million new cases and more than 900000 fatal cases every year, and its morbidity and mortality rank third and second in the world respectively $[5,6]$. COVID-19 pandemic poses a potential risk to cancer patients, that is, delayed diagnosis and treatment may lead to disease progression and survival shortening[7]. As the epidemic situation has been effectively controlled in most parts of China, the normal diagnosis and treatment order of colorectal cancer is gradually restored. It is very important to sum up the experience during the epidemic and to better carry out clinical work in the situation of regular epidemic prevention and control.

During the severe epidemic of COVID-19, the number of non-local patients coming to the hospital decreased due to the adoption of traffic restrictions and the strategy of reducing personnel mobility. On behalf of Group of Colorectal Surgery, Society of Surgery, Chinese Medical association, our team drafted and published a Chinese expert consensus on surgical diagnosis and treatment strategies for CRC patients during COVID-19 epidemic[8]. The flow chart of diagnosis and treatment of CRC during COVID-19 is shown in Fig. 1.

We emphasize the importance of multidisciplinary team (MDT) meetings in the formulation of diagnosis and treatment strategies for colorectal cancer patients (screening for COVID-19 infection was negative) during the epidemic. MDT can effectively integrate the advantages of multiple disciplines, more reasonable pre-treatment evaluation of patients, and strive to ensure that patients get effective treatment $[9,10]$, under the premise of minimizing the risk of COVID-19 infection during treatment.

During the COVID-19 epidemic, CRC patients inevitably have difficulties in hospitalization and delayed operation[11, 12]. We suggest that Neo-CRT should be given priority according to patients' condition, and radical surgery should be performed when epidemic remission. Both National Comprehensive Cancer Network (NCCN) and European Society for Medical Oncology (ESMO) guidelines recommend Neo-CRT, 
surgery and adjuvant chemotherapy as the gold standard treatment for locally advanced rectal cancer[13, 14]. The implementation of Neo-CRT can significantly benefit patients with locally advanced rectal cancer in reducing the local recurrence rate and improving the rate of sphincter preservation $[15,16] .15 \%$ of rectal cancer patients who underwent radical surgery after Neo-CRT could achieve complete remission of tumor pathology, that is, there was no tumor residue in the surgical specimens, and this part of the patients had a good prognosis[17]. According to Consensus on the Watch and Wait policy in rectal cancer patients after neoadjuvant treatment in China (2020 version), patients who are judged to be clinically complete remission or near clinical complete remission after Neo-CRT can directly enter the follow-up process of waiting and watch (W\&W)[18]. According to the actual situation during the epidemic period of COVID-19, patients can choose to adopt the non-operative treatment strategy of W\&W. Although neoadjuvant chemotherapy for patients with colon cancer is still in the exploratory stage, it is considered to have the advantages of reducing the primary tumor, killing micrometastases as soon as possible, reducing iatrogenic dissemination, increasing local drug concentration, and being conducive to the choice of postoperative chemotherapeutic drugs. the early results of previous studies have shown that the effective rate of neoadjuvant chemotherapy for colon cancer is good, the clinical remission rate and pathological remission rate are $68 \%$, and no patient has disease progression[19].

A multicenter cross-sectional study in China showed that 7.2\%(83/1 147)of CRC patients' surgery were affected by COVID-19 outbreak, and 3.4\% of patients underwent emergency surgery because of colorectal cancer complications[20]. In order to deal with the influence of COVID-19, China[8], Italy[21] and France[22] have all made some changes to the surgical treatment strategies of CRC. The principle of endoscopic treatment or radical surgery for patients with early CRC (CT1N0) and some locally advanced CRC (cT2-3N0) remains basically unchanged, but can be appropriately delayed; for patients with locally advanced CRC (CT 4) and patients with distant metastasis who can be resected by radical resection, elective surgery is performed after Neo-CRT; short-term neoadjuvant radiotherapy is recommended for rectal cancer (cT3-4N+). Emergency surgery should be considered for CRC patients with primary tumor bleeding (endoscopic or interventional therapy is ineffective), perforation and obstruction.

Based on the above treatment strategies for CRC, in our study, we can found that patients who received surgical treatment during the severe epidemic period are mainly in stage $\nabla$ and $\Downarrow$. More stage $\nabla$ patients underwent surgery in the period of remission. But for these stage $\otimes$ patients who choose to postpone surgical treatment, their long-term survival still needs follow-up observation. In our study, we found a special phenomenon that a higher proportion of patients had vascular and perineural invasion in SEG, this may be related to a higher proportion of patients in ECG had received preoperative Neo-CRT. In some CRC patients Neo-CRT can delay local tumor progression or even local regression[23, 24]. At the same time, in our study, the proportion of postoperative complications in SEG was higher, and most of these patients received preoperative Neo-CRT (5/6). Previous studies had suggested that preoperative Neo-CRT may lead to the incidence of postoperative complications increasing[25, 26]. Therefore, when considering the benefits of Neo-CRT during COVID-19 epidemic, attention should also be paid to the increased risk of postoperative complications. 
This study only included patients undergoing CRC surgery in a single center in Beijing, China. In view of the current epidemic of COVID-19 in the world, the public health resources, populace obedience and some aspects vary in different regions, for which some of our recommendations might be impractical to imitate. In addition, the sample size is limited, so the results of this study still need to be verified by multicenter and larger sample size studies. Furthermore, the data analysis of this study is lack of subgroup analysis, and the related results may have potential confounding factors.

\section{Conclusions}

Our study shows that colorectal surgery during COVID-19 is safe and feasible, and we hope that our strategy for diagnosis and treatment of colorectal cancer would be helpful for other surgeons. At the same time, due to the interference of serious epidemic situation to medical activities and more patients choosing preoperative neoadjuvant chemoradiotherapy, some patients delayed operation to the period of the epidemic remission, which may lead to the progress of tumor staging and increase the risk of postoperative complications, hoping to attract the attention of surgeons.

\section{Abbreviations}

BMI, body mass index; COVID-19, coronavirus disease 2019; CRC, colorectal cancer; CC, colon cancer; dMMR, deficient mismatch repair; ECG, epidemic remission group; ICTV: International Committee on Taxonomy of Viruses; MDT, multiple disciplinary treatment; M, mean; N, number; Neo-CRT, neoadjuvant chemoradiotherapy; pMMR, proficient mismatch repair; RC, rectal cancer; SEG, severe epidemic group; SD, standard deviation; W\&W, watch and wait strategy.

\section{Declarations}

\section{Ethics approval and consent to participate}

This study was approved by the Institutional Review Board of General Hospital of PLA(S2020-003-08).

\section{Consent to publish}

Not applicable.

\section{Availability of data and materials}

The datasets used and/or analysed during the current study are available from the corresponding author on reasonable request.

\section{Competing interests}

The authors declared no competing interests. 


\section{Funding}

None.

\section{Authors' contributions}

XHD and SYL conceived the analysis. YY and YXL extracted all data. YY, YXL and SYL undertook and refined the inclusion process. $Y Y$ and $Y X L$ co-wrote the paper. $Y Y$ and $S Y L$ undertook the statistical analyses. All authors contributed to and revised the final manuscript.

\section{Acknowledgements}

Not applicable.

\section{References}

1. Zhu N, Zhang D, Wang W, et al. A Novel Coronavirus from Patients with Pneumonia in China, 2019[J]. N Engl J Med. 2020;382(8):727-33.

2. Li Q, Guan X, Wu P, et al. Early Transmission Dynamics in Wuhan, China, of Novel CoronavirusInfected Pneumonia[J]. N Engl J Med. 2020;382(13):1199-207.

3. Liang W, Guan W, Chen R, et al. Cancer patients in SARS-CoV-2 infection: a nationwide analysis in China[J]. Lancet Oncol. 2020;21(3):335-7.

4. Wang D, Hu B, Hu C, et al. Clinical Characteristics of 138 Hospitalized Patients With 2019 Novel Coronavirus-Infected Pneumonia in Wuhan, China[J]. JAMA. 2020;323(11):1061-9.

5. Chen W, Zheng R, Baade PD, et al. Cancer statistics in China, 2015[J]. CA Cancer J Clin. 2016;66(2):115-32.

6. Bray F, Ferlay J, Soerjomataram I, et al. Global cancer statistics 2018: GLOBOCAN estimates of incidence and mortality worldwide for 36 cancers in 185 countries[J]. CA Cancer J Clin. 2018;68(6):394-424.

7. Wang H, Zhang L. Risk of COVID-19 for patients with cancer[J]. Lancet Oncol. 2020;21(4):e181.

8. He C, Li Y, Huang $X$, et al. How should colorectal surgeons practice during the COVID-19 epidemic? A retrospective single-centre analysis based on real-world data from China[J]. ANZ J Surg. 2020.

9. De Greef K, Rolfo C, Russo A, et al. Multisciplinary management of patients with liver metastasis from colorectal cancer[J]. World J Gastroenterol. 2016;22(32):7215-25.

10. van de Velde CJ, Boelens PG, Borras JM, et al. EURECCA colorectal: multidisciplinary management: European consensus conference colon \& rectum[J]. Eur J Cancer. 2014;50(1):1.e1-1.e34.

11. Loveday C, Sud A, Jones ME, et al. Prioritisation by FIT to mitigate the impact of delays in the 2-week wait colorectal cancer referral pathway during the COVID-19 pandemic: a UK modelling study[J]. Gut. 2020 . 
12. Torzilli G, Viganò L, Galvanin J, et al. A Snapshot of Elective Oncological Surgery in Italy During COVID-19 Emergency: Pearls, Pitfalls, and Perspectives[J]. Ann Surg. 2020;272(2):e112-112e117.

13. Petrelli F, Sgroi G, Sarti E, et al. Increasing the Interval Between Neoadjuvant Chemoradiotherapy and Surgery in Rectal Cancer: A Meta-analysis of Published Studies[J]. Ann Surg. 2016;263(3):458-64.

14. Recio-Boiles A, Hammad H, Howell K, et al. Locally Advanced Rectal Cancer Evaluation by Magnetic Resonance Imaging after Neoadjuvant Therapy on Decision Making: Cancer Center Experience and Literature Review[J]. J Gastrointest Cancer. 2020;51(1):254-9.

15. Perez K, Safran H, Sikov W, et al. Complete Neoadjuvant Treatment for Rectal Cancer: The Brown University Oncology Group CONTRE Study[J]. Am J Clin Oncol. 2017;40(3):283-7.

16. CSOCOC DATGFCCWG. Chinese Society of Clinical Oncology (CSCO) diagnosis and treatment guidelines for colorectal cancer 2018 (English version)[J]. Chin J Cancer Res. 2019;31(1):117-34.

17. Maas M, Nelemans PJ, Valentini V, et al. Long-term outcome in patients with a pathological complete response after chemoradiation for rectal cancer: a pooled analysis of individual patient data[J]. Lancet Oncol. 2010;11(9):835-44.

18. [Consensus on the Watch and Wait policy in rectal cancer patients after neoadjuvant treatment (2020 version)][J]. Zhonghua Wei Chang Wai Ke Za Zhi. 2020;23(1):1-9.

19. Liu F, Yang L, Wu Y, et al. CapOX as neoadjuvant chemotherapy for locally advanced operable colon cancer patients: a prospective single-arm phase II trial[J]. Chin J Cancer Res. 2016;28(6):589-97.

20. Yang Y, Gao ZY, Chen YK, et al. [Effects on the integrated treatment of colorectal cancer patients during COVID-19 epidemic in China: a cross-sectional study][J]. Zhonghua Wei Chang Wai Ke Za Zhi. 2020;23(8):795-800.

21. Di SS, Pata F, Gallo G, et al. Coronavirus pandemic and colorectal surgery: practical advice based on the Italian experience[J]. Colorectal Dis. 2020;22(6):625-34.

22. An $\mathrm{P}, \mathrm{Ye} Y$, Chen $\mathrm{M}$, et al. Management strategy of novel coronavirus (COVID-19) pneumonia in the radiology department: a Chinese experience[J]. Diagn Interv Radiol. 2020;26(3):200-3.

23. Dattani M, Heald RJ, Goussous G, et al. Oncological and Survival Outcomes in Watch and Wait Patients With a Clinical Complete Response After Neoadjuvant Chemoradiotherapy for Rectal Cancer: A Systematic Review and Pooled Analysis[J]. Ann Surg. 2018;268(6):955-67.

24. Dossa F, Acuna SA, Rickles AS, et al. Association Between Adjuvant Chemotherapy and Overall Survival in Patients With Rectal Cancer and Pathological Complete Response After Neoadjuvant Chemotherapy and Resection[J]. JAMA Oncol. 2018;4(7):930-7.

25. Glynne-Jones R, Wyrwicz L, Tiret E, et al. Rectal cancer: ESMO Clinical Practice Guidelines for diagnosis, treatment and follow-up[J]. Ann Oncol. 2018;29(Suppl 4):iv263.

26. Karoui M, Rullier A, Piessen G, et al. Perioperative FOLFOX 4 Versus FOLFOX 4 Plus Cetuximab Versus Immediate Surgery for High-Risk Stage II and III Colon Cancers: A Phase II Multicenter Randomized Controlled Trial (PRODIGE 22)[J]. Ann Surg. 2020;271(4):637-45. 


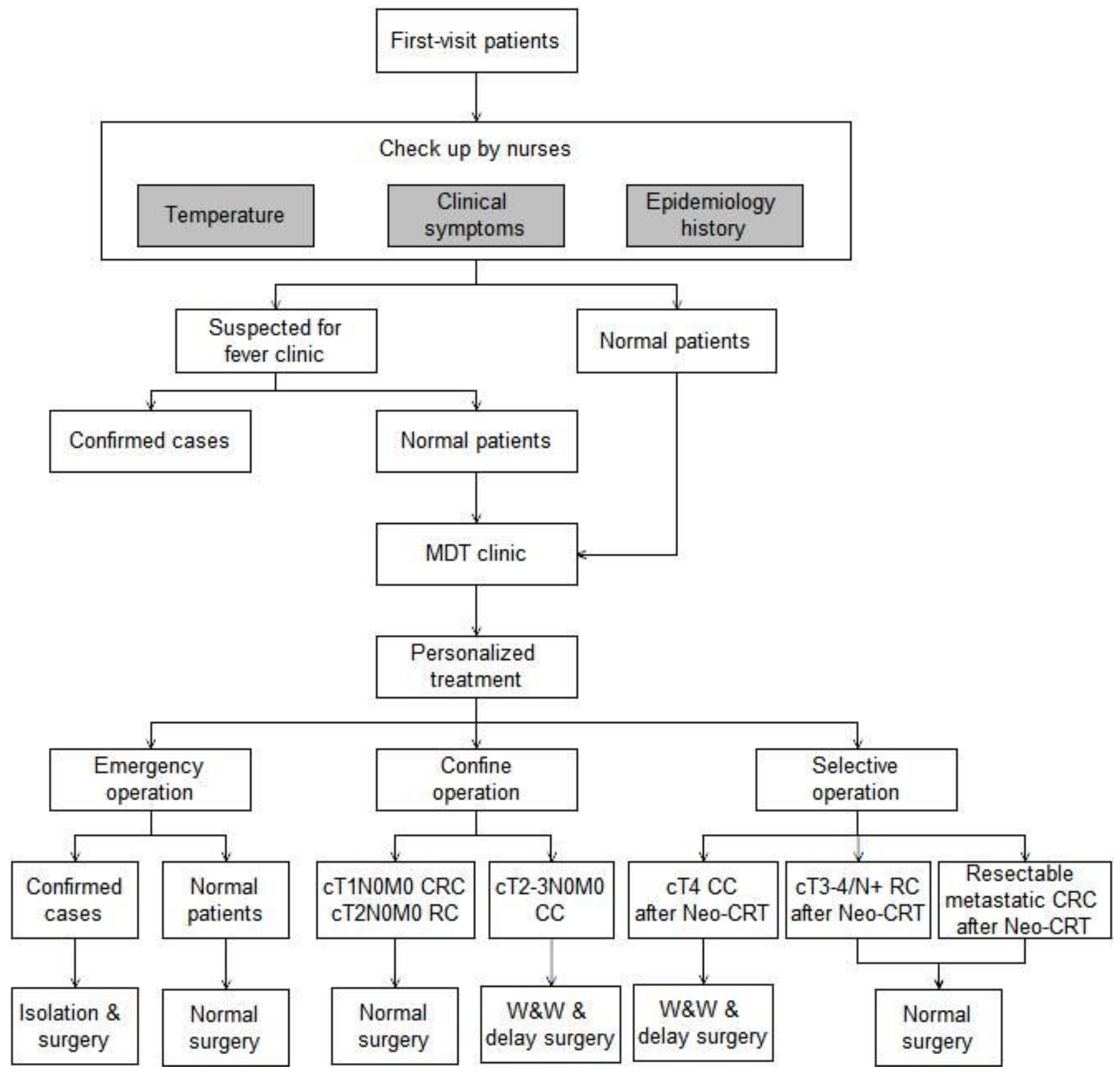

Figure 1

Flowchart of diagnosis and treatment for colorectal cancer patients during COVID-19 Legends: MDT, multiple disciplinary treatment; $\mathrm{CRC}$, colorectal cancer; $\mathrm{RC}$, rectal cancer; $\mathrm{CC}$, colon cancer; W\&W, watch and wait strategy; Neo-CRT, neoadjuvant chemoradiotherapy. 